2 counters." More interaction between mathematical probabilists and those applying probability to discrete problems will benefit both groups.

\title{
REFERENCES
}

1. D. E. Knuth, The art of computer programming, vol. 3, Addison-Wesley, Reading, MA, 1973.

2. F. P. Preparata and M. I. Shamos, Computational geometry, Springer-Verlag, New York, 1985.

3. J. S. Vitter and P. Flajolet, Analysis of algorithms and data structures, Handbook of Theoretical Computer Science, vol. A: Algorithms and Complexity, Ch. 9, North-Holland, Amsterdam, 1990, pp. 431-524.

David Aldous

UNIVERSITY OF CALIFORNIA AT BERKELEY

BULLETIN (New Series) OF THE

AMERICAN MATHEMATICAL SOCIETY

Volume 27, Number 2, October 1992

(C) 1992 American Mathematical Society

$0273-0979 / 92 \$ 1.00+\$ .25$ per page

The topology of torus actions on symplectic manifolds, by Michèle Audin. Birkhäuser-Verlag, Basel, Boston, and Berlin, 1991, 181 pp., \$64.00. ISBN 3-7643-2602-6, ISBN 0-8176-2602-6

Nowadays it seems to be widely admitted that a mathematical theory reaching its final stage often becomes almost a triviality. The main technical emphasis in such a theory is put on the language, i.e., the notions and definitions, which must be very precise, though often quite complex to cover various applications, and so developed that the proofs turn out simple and clear. A basic example here is the theory of differential forms with its highly elaborated language and very few simple, but fundamental results like Stoke's theorem and Poincaré's lemma. The theory of Hamiltonian actions did originally invent certain very useful notions, e.g., the momentum mapping, but also borrowed some technical tools (Morse theory, equivariant cohomology) from other fields. Now, after a decade of dramatic progress, many chapters of the theory have reached the final stage, and a perfect indication of this is the book by Michèle Audin.

\section{Convexity Theorem}

Perhaps the most famous and beautiful result that the theory of Hamiltonian torus actions is known for is the Atiyah-Guillemin-Sternberg convexity theorem [At] and [GS1]:

Let $(W, \omega)$ be a compact connected symplectic manifold equipped with a Hamiltonian action of a torus $T$. Then the image $P$ of the momentum mapping $J: W \rightarrow t^{*}$ is a convex polyhedron and all the preimages $J^{-1}(y), y \in \mathfrak{t}^{*}$ are connected.

The convexity theorem is a very advanced generalization of the classical result by Schur [S]: the main diagonals of $n \times n$ Hermitian matrices with fixed eigenvalues form a convex set in $\mathbb{R}^{n}$. The reader interested in the relation between these two results should consult the book or original papers [At, GS1].

Here I cannot resist the temptation to outline the proof, but first let us recall the definitions and notions we have used. A symplectic manifold is a manifold 
equipped with a closed 2-form $\omega$ which satisfies the nondegeneracy hypothesis: $\omega^{n} \neq 0$ anywhere on $W$. Here, $2 n=\operatorname{dim} W$. Examples: the cotangent bundle to a manifold and coadjoint orbits of a Lie group carry natural symplectic structures. Any function $h$ on $W$ gives rise to the vector field $\xi$ defined by the Hamilton equation $i_{\xi} \omega=d h$. The Hamiltonian $h$ is said to be periodic if $\xi$ generates a circle action on $W$; a circle action obtained in this way is called Hamiltonian. It is easy to see that the critical points of $h$ are precisely the fixed points of the action. The next bit of symplectic geometry we need is the Darboux theorem: a symplectic structure can be linearized near every point, i.e., it is locally diffeomorphic to the "standard" linear symplectic structure $\sum d p_{i} \wedge d q_{i}$ on $\mathbb{R}^{2 n}$. Moreover, it can be linearized along with a Hamiltonian action of the circle $T^{1}$ (the equivariant Darboux theorem): if $x \in W$ is a fixed point of the action then a neighborhood of $x$ is equivariantly diffeomorphic to a neighborhood of the origin in $\mathbb{R}^{2 n}$ equipped with the standard symplectic structure and the $T^{1}$-action given by the quadratic Hamiltonian $\sum a_{i}\left(p_{i}^{2}+q_{i}^{2}\right)$. This implies that $h$ is a Morse-Bott function (i.e., its critical set is a smooth submanifold and $d^{2} h$ is nondegenerate on the transversals to the critical set), the critical submanifolds of $h$ are even-dimensional (in fact, symplectic), and all the indexes are even. Now Morse theory tells us that all the levels of $h$ are connected (the convexity theorem for $T^{1}$-actions!) and, in particular, $h$ has a unique local maximum and minimum. If the critical points of $h$ are isolated then $h$ is a perfect Morse function.

Consider now a Hamiltonian action of the torus $T$ on $W$ with the momentum mapping $J$. Such an action can also be linearized near a fixed point. This argument implies that:

(i) The image $J(P)$ is "locally convex": for any $x \in W$ there exists a neighborhood $U$ of $x$ and $V$ of $y=J(x)$ such that $J(U)=C_{x} \cap V$, where $C_{x}$ is an affine convex polyhedral cone in $\mathfrak{t}^{*}$ with the vertex at $y$. (To prove this it is enough to linearize the action of $\operatorname{Stab}_{x}$.)

(ii) Everything we said about a periodic Hamiltonian remains correct for a quasi-periodic one, i.e., for $h \in C^{\infty}(W)$ such that the flow of $\xi$ topologically generates a torus action on $W$. In particular, for any linear function $\pi$ on $\mathfrak{t}^{*}$ the function $\pi \circ J$ has a unique local minimum.

(iii) For every $x \in W$ we have $P \subset C_{x}$. In fact, if $P$ is not contained in $C_{x}$, then one could find a linear function $\pi$ such that $\pi \circ J$ has at least two local minima. But this contradicts (ii)!

Finally, by (i) and (iii) the image $P$ must be a convex polyhedron and, moreover, $P$ coincides with the intersection of all the cones $C_{x}$. Arranging this argument in a more elaborate fashion one can prove that the "fiber" of $J$ is connected.

\section{Equivariant cohomology and the DuistermaAt-Heckman formula}

The stationary phase principle takes an especially simple and explicit form for a periodic Hamiltonian $h$. Namely, it was proved by Duistermaat and Heckman [DH] that if all the critical points $x$ of $h$ are isolated then we have the exact formula

$$
\int_{W} e^{-\sqrt{-1} h t} \frac{\omega^{n}}{n !}=\frac{1}{(\sqrt{-1} t)^{n}} \sum_{x} \frac{e^{-\sqrt{-1} h(x) t}}{e_{x}},
$$


where the integer number $e_{x}$ is assigned to a critical point $x$ as follows. Every $x$ is a fixed point of the $T^{1}$-action given by $h$; thus, as before we can linearize the action near $x$ and so write $h=h(x)+\sum a_{i}\left(p_{i}^{2}+q_{i}^{2}\right)$ in some local coordinates $p_{i}, q_{i}$ near $x$. Then we set $e_{x}=a_{1} a_{2} \cdots a_{n}$. Note that $e_{x}$ is an integer because $h$ is periodic and so all coefficients $a_{i}$ must be integers.

$A$ very simple and enlightening proof of this result is due to Atiyah and Bott $[\mathrm{AB}]$ and Berline and Vergne [BV]. The key point of the proof is to treat the left-hand side as the integral of certain equivariant cohomology class. Let $T=T^{1}$. Following [AB] we identify the $T$-equivariant cohomology $H_{T}^{*}(W)$ with the cohomology of the equivariant de Rham complex. This complex is just the graded ring $\Omega_{T}^{*}(W)[u]$ equipped with the differential $D=d+i_{\xi} u$, where $u$ is an "independent variable," $\operatorname{deg} u=2$, and $\Omega_{T}^{*}(W)$ is the ring of $T$-invariant differential forms. For example, if the action is trivial: $\xi=0$, then $H_{T}^{*}(W)=H^{*}(W) \otimes \mathscr{R}$, where $\mathscr{R}=\mathbb{R}[u]$. Note also that $\omega$ is not equivariantly closed, but its "extension" $\omega-h \cdot u$ is.

Pick an equivariant form $\sigma \in \Omega_{T}^{*}(W)[u]$ and expand it as $\sum \sigma_{i} f_{i}(u)$, where $f_{i} \in \mathscr{R}$ and $\sigma_{i}$ is a differential form of degree $i$. Now it is natural to set $\int_{W} \sigma=f_{2 n} \int_{W} \sigma_{2 n}$. The localization theorem (see [AB] or the book) says that the integral of $\sigma$ depends only on the restriction of $\sigma$ to the fixed point set $W^{T}$ :

$$
\int_{W} \sigma=\sum_{x \in W^{T}} \frac{\sigma_{0}(x) f_{0}(u)}{e_{x} u^{n}}
$$

To prove (1) we apply (2) to $\sigma=\exp (\omega-h \cdot u)$ and set $u=\sqrt{-1} t$.

Among various applications of (2) we would like to mention just one more, namely, the relation

$$
\sum_{x \in W_{T}} \frac{1}{e_{x}}=0,
$$

which, as well as (2), holds for a symplectic, i.e., $\omega$-preserving, or even almostcomplex $T$-action. To prove it one has to substitute $\sigma=1$ in (2). For a Hamiltonian action (3) also follows from (1) [DH].

The original proof of (1) is based on another argument, though it is also relevant to equivariant cohomology. Namely, the right-hand side of (1), regarded as a function of $t$, is the Fourier transform of the function $f$ such that for any regular value $c$ of $h$ we set $f(c)$ equal to the volume of the level $M_{c}=\{h=c\}$, i.e., $f(c)$ is the integral of $\omega^{2 n} / d h$ over $M_{c}$. According to Duistermaat and Heckman [DH], $f$ is a piecewise polynomial function. To see this we equivariantly identify all the levels $M_{c}, c \in I=\left(c_{0}, c_{1}\right)$, where the interval $I$ does not contain critical points of $h$. Thus the reduced symplectic structure $\omega_{c}$ on $B_{c}=M_{c} / T$, becomes a function of $c$. A straightforward calculation shows that this function is linear (i.e., affine), so $f(c)=\int_{B_{c}} \omega_{c}^{n-1}$ must be polynomial on $I$.

Coming back to equivariant cohomology we observe that $H_{T}^{*}\left(M_{c}\right)=$ $H^{*}\left(B_{c}, \mathbb{R}\right)$ because the $T$-action on $M_{c}$ is locally free and the equivariant cohomology is taken over $\mathbb{R}$. It turns out that the derivative $\chi=d\left[\omega_{c}\right] / d c$ has a nice topological interpretation. Namely, the cohomology class $\chi$ is the so-called Euler class of the locally free action on $M_{c}$ (independent of $c \in I$ ). 
For example, if the action is free, then $\chi$ is just the Euler class of the circle bundle $M_{c} \rightarrow B_{c}$. Motivated by this simple analysis, we come to the question of what happens to $B_{c}, \chi$, and $\omega_{c}$ when $c$ goes through a critical value of $h$. The book under review contains a detailed account on this matter (see also [M, G] and, in particular, [GS2]).

As we see equivariant cohomology is a useful and powerful tool to be used in the theory of Hamiltonian actions. But what makes it especially attractive is that $H_{T}^{*}(W)$ is easy to calculate. Namely, for a Hamiltonian action $H_{T}^{*}(W)$ is isomorphic to $H^{*}(W) \otimes \mathscr{R}$ as an $\mathscr{R}$-module. In other words the cohomology does not feel the action at all, i.e., it behaves as if the action were trivial. This result is apparently due to Kirwan [K1].

The proof is especially simple for a circle action with isolated fixed points. Analyzing the Morse complex of $h$ it is not hard to show that any closed $T$ invariant $k$-form $\sigma_{k}$ admits a $D$-closed "extension" $\sigma=\sigma_{k}+\sigma_{k-2} u+\sigma_{k-4} u^{2}+$ $\cdots$, where $\sigma_{i} \in \Omega_{T}^{i}(W)$. Moreover, the extension $\sigma$ can be made canonical if, for example, we employ the Hodge theory. Thus we have an $\mathbb{R}$-linear monomorphism $H^{*}(W) \rightarrow H_{T}^{*}(W)$, which, being $\mathscr{R}$-linear extended, gives rise to the desired isomorphism $H^{*}(W) \times \mathscr{R} \rightarrow H_{T}^{*}(W)$.

Unfortunately this simple method does not work that well when the fixed points are not isolated. However, applying a more advanced technique, one can prove the general case literally in a few lines. Recall that there exists a spectral sequence with $E_{2}^{*}=H^{*}(W) \otimes \mathscr{R}$ converging to the equivariant cohomology and thus $\mathrm{rk}_{\mathscr{R}} H_{T}^{*}(W) \leq \operatorname{dim} H^{*}(W)$. On the other hand, by the Borel localization theorem [B] (see also the book or [AB] for a modern treatment) $\operatorname{rk}_{\mathscr{R}} H_{T}^{*}(W)=\operatorname{dim} H^{*}\left(W^{T}\right)$. Finally, since $W^{T}$ is the critical set of a quasi-periodic Hamiltonian, $\operatorname{dim} H^{*}\left(W^{T}\right) \geq \operatorname{dim} H^{*}(W)$ and, as a consequence, $\mathrm{rk}_{\mathscr{R}} E_{2}^{*}=\mathrm{rk}_{\mathscr{R}} H_{T}^{*}(W)$. This only can happen when the spectral sequence degenerates and the $E_{2}$-term coincides with the equivariant cohomology. A rather formal argument shows that the same is true for a Hamiltonian action of any compact group. Moreover, this result (properly reformulated), as well as the Duistermaat-Heckman formula, remains correct for some symplectic actions $[\mathrm{G}]$.

\section{MORE ABOUT THE BOOK}

The book by Michèle Audin appears to be a perfect self-contained introduction into the theory of Hamiltonian torus actions. The first two chapters contain some preliminaries on compact group actions on manifolds (e.g., the slice theorem) and symplectic geometry. The topics we have discussed are covered in detail in Chapters 3 and 5, respectively, together with all the necessary technique (Morse theory and equivariant cohomology). In Chapter 5 the reader will be pleased to find a simple and intuitive definition of equivariant cohomology via the equivariant de Rham complex, which complements the standard approach based on formal, though useful and very powerful, algebraic topology constructions.

Chapter 4 is entirely devoted to the Hamiltonian actions on compact 4manifolds, in particular, circle actions. Such actions occupy a very special place in the theory, because, on the one hand, the first nontrivial examples are among them, and on the other hand, these actions can be effectively described [Au]. Namely, every such action can be obtained from one of a small number of basic 
examples by a sequence of blow-ups. Another interesting result we have in this dimension is that according to a theorem of McDuff [M] a symplectic circle action on a compact connected 4-manifold is Hamiltonian if and only if it has fixed points. Proof $[G]$ : Consider a non-Hamiltonian action; for the sake of simplicity we assume that the form $\alpha=i_{\xi} \omega$ is integral. Then the Morse theory argument shows that all the critical points $x$ of $\alpha$ are isolated and have index 2 , thus $e_{x}<0$. By (3) this is possible only if $W^{T}=\varnothing$. Conversely, it is clear that a Hamiltonian action does have fixed points, namely, critical points of a Hamiltonian.

The last chapter concerns toric varieties, which appear in this context as symplectic manifolds with the biggest Abelian compact group of symmetries. In other words a compact symplectic $2 n$-manifold is toric if it admits a Hamiltonian $T^{n}$-action. (Note that here toric manifolds carry symplectic rather than a complex structure.) Due to Delzant [D] such a manifold is uniquely determined by the image $P$ of the momentum mapping. Thus the topology of the manifold and the action can be recovered from the combinatorics of the polyhedron $P$. Reading this relatively elementary chapter requires considerable effort, in part, because some of the results are unclear or even incorrectly stated. (In particular, this concerns $\S 3$.) Hence I hardly can recommend it as an introduction in toric varieties. On the other hand, the approach developed here, in certain aspects, is much easier than the standard treatment of toric varieties. Thus I believe that experts will find the material presented in Chapter 6 interesting and very useful.

The book under review is by no means a monograph but rather a textbook. In other words Topology of torus actions on symplectic manifolds does not intend to cover the entire field, and some, even important, results are just briefly mentioned. Among such results are, for instance, the calculation of equivariant cohomology (outlined in the second section of this review) and the deep convexity theorem for non-Abelian groups due to Kirwan [K2]. (As the title indicates, the book is on torus actions.)

The book is written in an elegant and informal style, but not at the cost of details, and with a care about technicalities. Numerous, often very instructive, exercises and examples, as well as excursions into related areas, give the reader a chance to learn not only the theory and applications of Hamiltonian torus actions, but what may even be more important, its place in the complex conglomerate of modern mathematics.

\section{ACKNOWLEDGMENTS}

I am very grateful to A. Givental, Y. Karshon, Z. Reichstein, and S. Tolman for help in preparing this review.

\section{REFERENCES}

[At] M. Atiyah, Convexity and commuting Hamiltonians, Bull. London Math. Soc. 14 (1982), $1-15$.

[AB] M. Atiyah and R. Bott, The moment map and equivariant cohomology, Topology 23 (1984), $1-28$.

[Au] M. Audin, Hamiltoniens périodique sur les variétés symplectique compactes de dimension 4, Géométrie symplectique et mécanique, Proceedings 1988 (C. Albert, ed.), Lecture Notes in Math., vol. 1416, Springer-Verlag, Berlin and New York, 1990. 
[BV] N. Berline and M. Vergne, Zéros d'un champ de vecteurs et classes caractéristiques équivariantes, Duke Math. J. 50 (1983), 539-549.

[B] A. Borel et al., Seminar on transformation groups, Ann. of Math. Stud., vol. 46, Princeton Univ. Press, Princeton, NJ, 1961.

[D] T. Delzant, Hamiltoniens périodiques et images convexes de l'application moment, Bull. Soc. Math. France 116 (1988), 315-339.

[DH] J. J. Duistermaat and G. J. Heckman, On the variation in the cohomology of the symplectic form of the reduced phase space, Addendum, Invent. Math. 69 (1982), 259-269, 72 (1983), 153-158.

[G] V. L. Ginzburg, Some remarks on symplectic actions of compact groups, Math. Z. (to appear, 1992).

[GS1] V. Guillemin and S. Sternberg, Convexity properties of the moment mapping, Invent. Math. 67 (1982), 491-513.

[GS2] _ Birational equivalence in symplectic geometry, Invent. Math. 97 (1989), 485-551.

[K1] F. Kirwan, Cohomology of quotients in symplectic and algebraic geometry, Math. Notes, vol. 31, Princeton Univ. Press, Princeton, NJ, 1984.

[K2] Convexity properties of the moment mapping, III, Invent. Math 77 (1984), 547-552.

[M] D. McDuff, The moment map for circle actions on symplectic manifolds, J. Geom. Phys. 5 (1988), 149-161.

[S] I. Schur, Über eine Klasse von Mittelbildungen mit Anwendungen auf der Determinantentheory, Sitzungsber. Berlin. Math. Ges. 22 (1923), 9-20.

VIKTOR L. GINZBURG

STANFORD UNIVERSITY

E-mail address: ginzburg@cauchy.stanford.edu

BULLETIN (New Series) OF THE

AMERICAN MATHEMATICAL SOCIETY

Volume 27, Number 2, October 1992

(C) 1992 American Mathematical Society

$0273-0979 / 92 \$ 1.00+\$ .25$ per page

The energy method, stability, and nonlinear convection, by Brian Straughan, Springer-Verlag, New York, 1992, xii+242 pp., \$49.80. ISBN 0-387-97658-2

Suppose that $f(\cdot)$ is a nonnegative, differentiable function defined on $I=$ $\left[t_{0}, \infty\right)$ and that $f^{\prime}(t) \leq a(t) f(t)$ for some locally integrable function $a(\cdot)$ on $I$. Then

$$
0 \leq f(t) \leq f(\tau) \exp \left(\int_{r}^{t} a(\eta) d \eta\right)
$$

for $t_{0} \leq \tau \leq t<+\infty$. This observation is called Gronwall's Inequality. If $\int^{+\infty} a(\eta) d \eta=-\infty$, then $\lim _{t \rightarrow+\infty} f(t)=0$.

The book under review is concerned with applications of this simple principle to questions of stability of solutions of problems in fluid dynamics. These problems are not simple.

The application of this principle to the fluid dynamical problems discussed in Straughan's book is often called the Energy Method and is closely related to the Lyapunov method. These problems have a rich mathematical and physical structure and are among the most important problems in applied mathematics. We shall mention a few of them later in this review.

The underlying feature of all of these problems is that the fluid motion itself is the primary mechanism which drives the transport of various physical quantities such as momentum, temperature, salinity, etc. This transport by the 